\title{
Glandular odontogenic cyst of the mandible: a case report and immunohistochemical study
}

\author{
Megumi Yokoyama, Hiroyuki Okada, Miyuki Morikawa, Hirotsugu Yamamoto \\ Department of Oral Pathology, Nihon University School of Dentistry at Matsudo, Matsudo, Japan
}

Abstract: Glandular odontogenic cyst (GOC) is a relatively rare jaw cyst. We report a case of GOC arising in the retromolar region, and associated with an impacted third molar of the mandible in a 37-year-old man. The surgical specimen was investigated histopathologically and immunohistochemically. Immunohistochemically, cytokeratin 19 was positive in the whole layer of the cystic lining as well as in the duct-like parts. The lining epithelia showed labeling indices of 7.2 for PCNA, 3.2 for topoisomerase IIa, and 11.6 for $\mathrm{Ki}-67$, respectively. These results indicated odontogenic and rather proliferative nature of GOC.

[Oral Med Pathol 2009; 13: 111-114 doi: 10.3353/omp.13.111]

Key words: glandular odontogenic cyst, immunohistochemistry, proliferative activity

Correspondence: Hirotsugu Yamamoto, Department of Oral Pathology, Nihon University School of Dentistry at Matsudo 2-870-1, Sakaecho-Nishi, Matsudo, Chiba 271-8587, Japan

Phone \& fax: +81-47-360-9335, E-mail: yamamoto.hirotsugu@nihon-u.ac.jp

\section{Introduction}

Glandular odontogenic cyst (GOC) is an uncommon jawbone cyst, which was first described in 1988 by Gardner et al. (1). It was listed in the second version of the WHO Histological Typing of Odontogenic Tumours as "glandular odontogenic cyst" or "sialo-odontogenic cyst" (2), although the WHO 2005 no longer lists this cyst (3). Since there have been only a limited number of reports of GOC, its histopathogenesis and clinical characteristics are still largely unknown. However, its unique histological features have raised several questions concerning the origin, classification, and terminology (4).

Histopathologically, the cyst wall of GOC is lined by non-keratinized stratified squamous epithelium of relatively uniform thickness exhibiting a flat interface with the subjacent stroma. The superficial layer of the epithelium consists of eosinophilic cuboidal cells that are sometimes ciliated. Duct-like (glandular or pseudo-glandular) structures containing PAS-positive material in their lumina are recognized within the lining epithelium (5).

We report a case of GOC with features of dentigerous cyst, whose lining epithelia were shown to have immunohistochemical profiles suggesting an odontogenic origin and proliferative characteristics. We also reviewed the literature.

\section{Case report}

A 37-year-old man was referred to Nihon University Hospital, School of Dentistry at Matsudo, with a chief complaint of an uncomfortable feeling at the mandibular left molar region. The patient's medical history was insignificant. Panoramic examination showed a well-defined radiolucency around the impacted tooth crown (Fig. 1). Computed tomography $(\mathrm{CT})$ revealed a $1.7 \times 1.5 \mathrm{~cm}$ unilocular welldefined low-density area showing a cystic lesion which continued to the impacted tooth crown. Extirpation of the lesion and the impacted tooth was performed under local anesthesia using $2 \%$ lidocaine and the clinical diagnosis of dentigerous cyst (DC). Macroscopically, the extirpation material consisted of an irregular, cystic-shaped, grayishbrown lesion measuring $1.6 \times 1.4 \times 1.0 \mathrm{~cm}$. There have been no signs of recurrence after the operation.

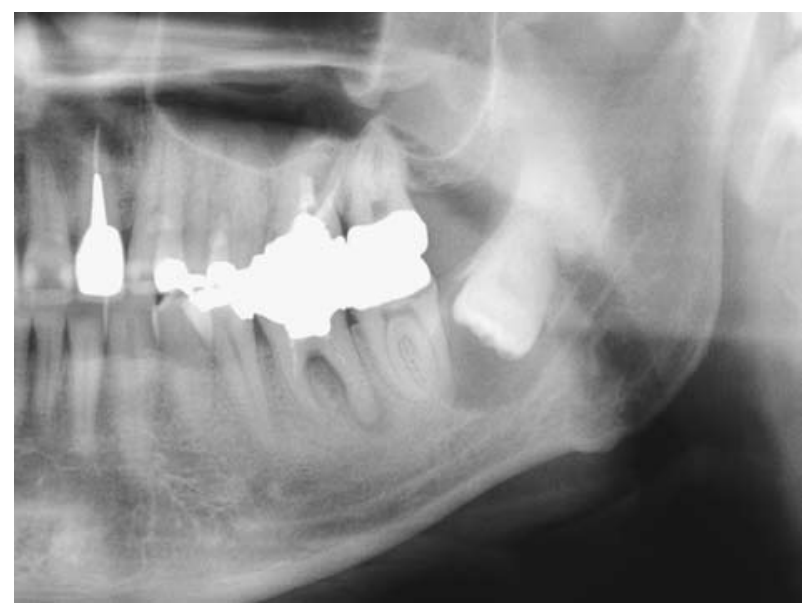

Fig. 1. A panoramic radiograph of glandular odontogenic cyst arising in the mandible of a 37-year-old man. The lesion was represented as a well-defined unilocular radiolucency around the crown of the impacted left third molar tooth. 


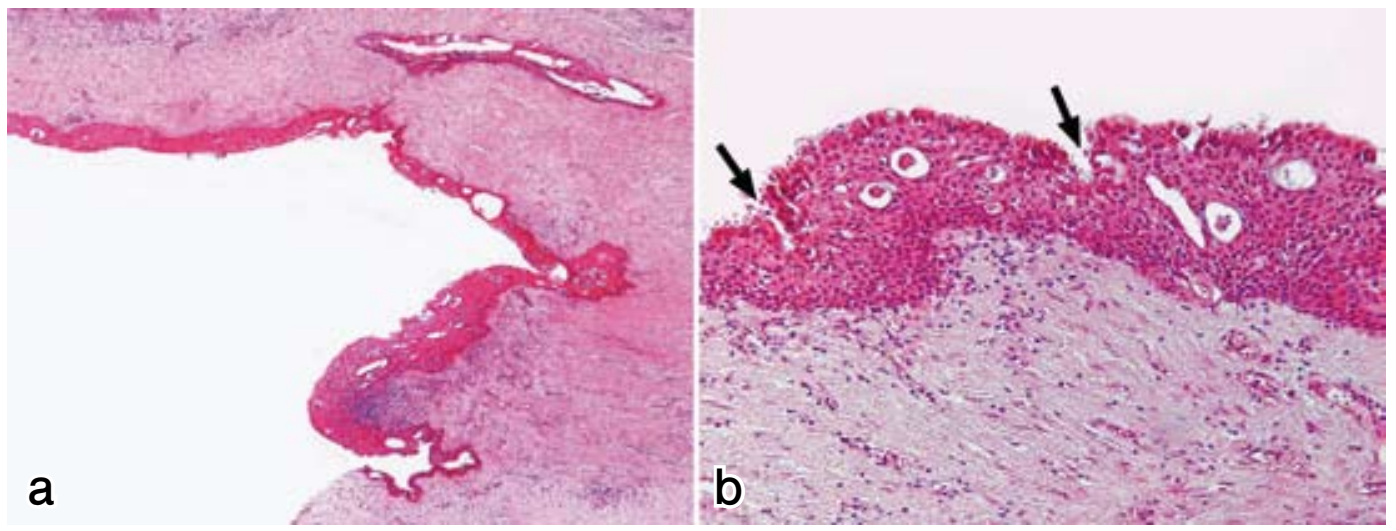

Fig. 2. Histopathological findings of glandular odontogenic cyst of the mandible. (a) Low power view of the cyst wall; (b) High power view of the cystic lining. Hematoxylin and eosin stain, (a) $\times 40$; (b) $\times 400$. The cystic spaces were lined by non-keratinized stratified squamous epithelium having an irregular surface. Some microcystic and duct-like spaces were recognized in the cystic wall (a). Superficial layer of epithelium consisted of cuboidal or low-columnar cells. Invaginations (b, arrows) were recognized. Intraepithelial microcystic/ductal structures contained mucous materials (b).

\section{Histopathological findings}

The cystic spaces were lined by non-keratinized stratified squamous epithelium which varied in thickness (Fig 2a). Numerous intraepithelial micro cystic/ductal structures lined by cuboidal cells, and partly with mucous cells, were recognized in the wide areas, and mucous materials were frequently seen (Fig. 2b). The superficial layer of the epithelium consisted of cuboidal or low-columnar cells with mucous cells that made the surface irregular (Fig. 2b). Invagination from the surface was also observed (Fig. 2b). Mucous cells were stained positively with combined alcian blue (AB)-periodic Schiff (PAS) (Fig. 3a) and mucicarmine (Fig. 3b). Mucous materials in the microcystic/ductal structures were also stained with PAS/AB (Fig. 3a). Hyaline body and small calcified materials were observed in the epithelium in parts. Subepithelially, mild lymphoid infiltration, expansion of microvessels, Russell bodies, myxoid degeneration, edema and hemorrhage were partly recognized in the fibrous connective tissue.

\section{Immunohistochemical findings}

Immunohistochemical staining was carried out with the dextran polymer method (Envision kit, Dako, Glostrup, Denmark). The primary antibodies against proliferating cell nuclear antigen (PCNA; clone PC10, diluted at 1: 100, Dako), topoisomerase II $\alpha$ (Ki-S1, 1: 50, Dako), Ki-67
(MIB-1, 1: 50, Dako), cytokeratin 18 (CK18, DC10, 1: 50, Dako) and cytokeratin 19 (CK19, RCK108, 1: 100, Dako) were used. As positive control for cytokeratin, tumor cells of pleomorphic adenoma and surrounding acinar and duct cells of the parotid gland were used. For proliferative activity, average positive cell rate (labeling index: LI) was calculated after analyzing about 500 to 1,000 cells in five fields at $\times 400$ magnification. On GOC, the expression of CK19 was moderately positive in the basal cell layer and slightly positive in the parabasal and surface layers and duct forming cells (Fig. 4a). CK18 was moderately positive in the surface layer and the duct forming cells in parts, and slightly positive in the parabasal layer, but CK18 was not positive in the basal cell layer (Fig. 4b). As a positive control, CK18 and CK19 were localized in duct cells of the parotid gland and duct forming cells of pleomorphic adenoma. CK18 was also expressed in acinar cells of the parotid gland. Positive cells for markers of proliferative activity were observed mainly in basal and parabasal layers. The LI of PCNA (Fig. 5a), topoisomerase II $\alpha$ (Fig. 5b), and Ki-67 (Fig. 5c) were 7.2, 3.2 and 11.6 , respectively.

\section{Discussion}

GOC is an uncommon lesion which was first described in 1988; its frequency rate is only 0.012 (6) to $1.3 \%$ (7) of all jaw cysts. At Nihon University Hospital, Dentistry at Matsudo, the rate has been $0.028 \%$. It was reported that

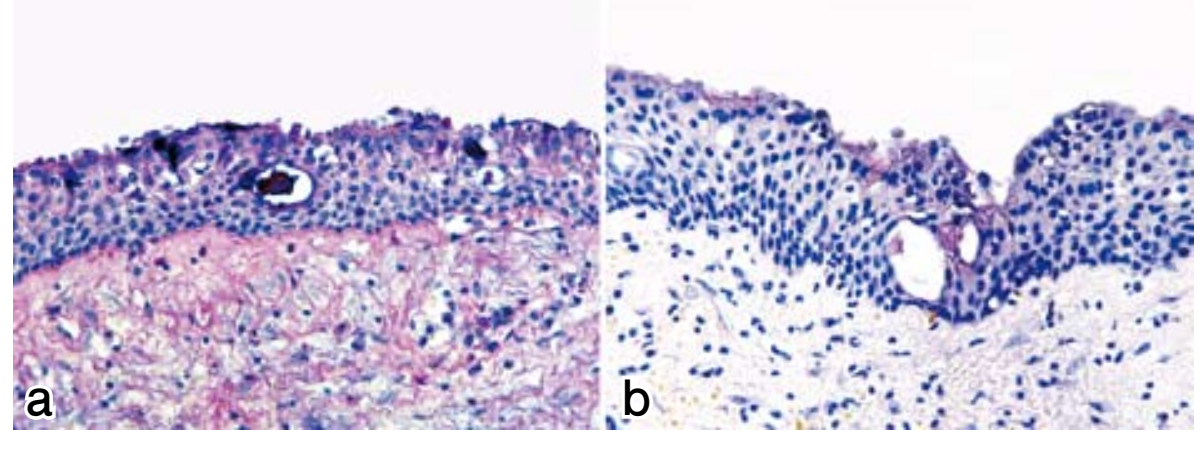

Fig. 3. Histochemical findings of lining epithelia of glandular odontogenic cyst of the mandible. (a) PAS/ $\mathrm{AB}$ stain; (b) mucicarmine stain. High power view of the cystic lining. $\times$ 400. Mucous cells and mucous materials are stained in blue by PAS/AB (a). Mucous cells forming duct-like structures as well as in the superficial layer were also positive for mucicarmine (b). 


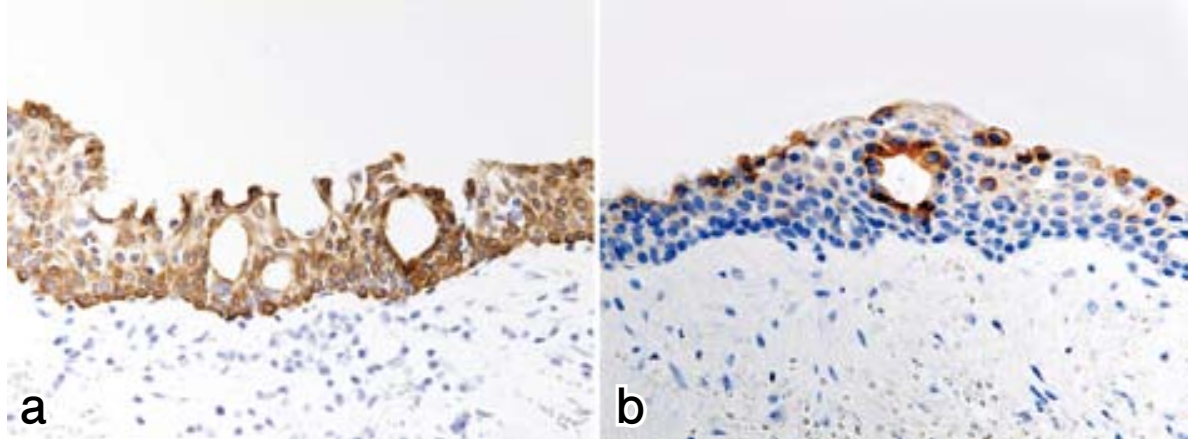

Fig. 4. Immunohistochemical findings for keratin subtypes in lining epithelia of glandular odontogenic cyst of the mandible. Immunoperoxidase stains for cytokeratin (CK) 19 (a) and CK18 (b), hematoxylin counterstain, $\times$ 400. CK19 was positive in the whole layer of the lining epithelia (a), while CK 18 was immunolocalized in the cells in the surface to parabasal layers including those forming duct-like structures (b).

GOC had a predilection for males and for middle aged patients, and that the mandible, especially the anterior mandible, was affected more often than the maxilla (8). Radiographically, the GOC is usually localized intraosseously and may appear as a unilocular or multilocular radiolucent lesion with well-defined borders (8). Recurrence is often seen. GOC has the potential aggressive behavior of a lesion (1). Clinical and radiographic findings of GOC can vary, and these finding are often not pathognomonic (9). Histologically, GOC shows certain characteristics, including non-keratinized and stratified epithelium. Thickened epithelial segments are seen within the lining epithelium, which may also display "epithelial swirls" but do not show any nuclear polarization. These swirls may also be seen in lateral periodontal cysts (LPC), justifiably pointing to the odontogenic nature of these cysts. The epithelial lining also occasionally contains eosinophilic cuboidal cells which may or may not be ciliated. Mucous cells and mucin pools are identified and result in a glandular appearance. These pools are stained by PAS/AB and show mucicarmine positive (9).

Intraepithelial duct-like structures have been also noted in other odontogenic cysts (10). Therefore, in the present case, the differential diagnosis from dentigerous cyst is important in terms of clinical and radiographical features. In the present study, cuboidal cells at the surface and their invagination were recognized. Duct-like structures were observed in the wide areas and they frequently contained PAS positive mucous materials in the lumen. Based on these histopathological features, the present case was diagnosed as GOC.

The histogenesis of GOCs remains uncertain but there are two theories of the origin of GOC; odontogenic and glandular origins. Most researchers believed that they originated from odontogenic epithelium (8). Important evidence in support of such a concept is the morphology of the epithelium. GOC arising in tooth-bearing areas led to the suggestion of an odontogenic epithelial origin, while histopathologically salivary components were noted. The thin, cuboidal or columnar epithelium is reminiscent of reduced enamel epithelium. The plaque-like or spherical structures sometimes are also seen in odontogenic lesions such as LPC, gingival cyst of adults and rarely in DC. Intraepithelial duct-like areas and mucous-producing cells with or without cilia have been ubiquitously reported in odontogenic lesions. The differential diagnosis among GOC, LPC and DC is important, because they have similar clinical (and histological) characteristics. This clinical and histological similarity is one of the most important pieces of evidence suggesting GOC is of odontogenic origin.

In this study, the whole layer of GOC lining epithelia showed immunopositivity to CK19, which has been shown to be specific to odontogenic epithelial cells. On the other hand, the basal layer of GOC linings was not positive to CK 18 , which has been known to be expressed in glandular epithelia. These results indicate that the origin of GOC linings was not ectopic salivary gland epithelium but odontogenic epithelium. It was also suggested that duct forming and mucus secreting cells might transform from odontogenic epithelium in the process of its differentiation.

There have been only a few reports about the proliferative activity on GOC. It was reported that the LI of Ki-67 (4.4) on GOC was significantly higher than that on radicular cyst

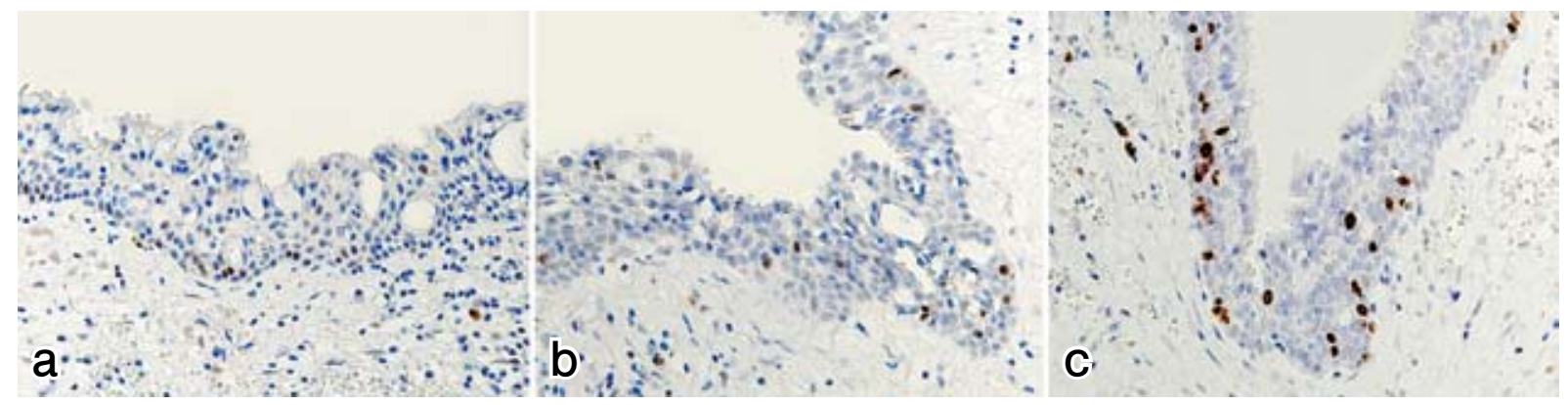

Fig. 5. Immunohistochemical findings for cell growth in lining epithelia of glandular odontogenic cyst of the mandible. Immunoperoxidase stains for proliferating cell nuclear antigen (PCNA) (a), topoisomerase II $\alpha(\mathbf{b})$, and Ki-67 (c), hematoxylin counterstain, $\times 400$. PCNA positive $(+)$ cells were recognized mainly in the basal and parabasal layers (a). Topoisomerase II $\alpha+$ cells were scattered in the lower half of the lining epithelium (b). Ki-67+ cells were frequently recognized in the basal to parabasal layers (c). 
with mucous metaplasia (RCM) (3.7), and that the LI of PCNA (19.0) on GOC was almost the same as that of RCM (18.6) (11). Another paper noted that the LI of Ki-67 (2.0) on GOC was lower than that on DC (9.0) (12). In the present study, GOC showed high proliferative activity: the LI of GOC was PCNA (7.2), Topoisomerase II $\alpha$ (3.2) and Ki-67 (11.6). Proliferative activity is a useful marker for revealing the biological behavior associated with recurrence rate of tumors, cysts, and various grades of malignant tumor. Further data concerning the proliferative activity of GOC is expected.

\section{References}

1. Gardner DG, Kessler HP, Morency R, Schaffner DL. The glandular odontogenic cyst: an apparent entity. J Oral Pathol 1988; 17: 359-66.

2. Kramer IRH, Pindborg JJ, Shear M. Histological typing of odontogenic tumours. Springer-Verlag, Berlin, 1991; 38.

3. Barnes L, Everson JW, Reichart P, Sidransky D. Pathology and Genetics of Head and Neck Tumours. World Health Organization Classification of Tumours. IARC Press, Lyon, 2005; 283-327.

4. Ide F. A note on the history and terminology of glandular odontogenic cyst. Oral Med Pathol 2007; 12: 101-2.

5. de Sousa SO, Cabezas NT, de Oliveira PT, de Araújo VC.
Glandular odontogenic cyst: report of a case with cytokeratin expression. Oral Surg Oral Med Oral Pathol Oral Radiol Endod 1997; 83: 478-83.

6. Magnusson B, Göransson L, Odesjö B, Gröndahl K, Hirsch JM. Glandular odontogenic cyst. Report of seven cases. Dentomaxillofac Radiol 1997; 26: 26-31.

7. van Heerden WF, Raubenheimer EJ, Turner ML. Glandular odontogenic cyst. Head Neck 1992; 14: 316-20.

8. Shen J, Fan M, Chen X, Wang S, Wang L, Li Y. Glandular odontogenic cyst in China: report of 12 cases and immunohistochemical study. J Oral Pathol Med 2006; 35: 175-82.

9. Sittitavornwong S, Koehler JR, Said-Al-Naief N. Glandular odontogenic cyst of the anterior maxilla: case report and review of the literature. J Oral Maxillofac Surg 2006; 64: 740-5.

10. Ide F, Shimoyama T, Horie N. Glandular odontogenic cyst with hyaline bodies: an unusual dentigerous presentation. $J$ Oral Pathol Med 1996; 25: 401-4.

11. Kaplan I, Anavi Y, Manor R, Sulkes J, Calderon S. The use of molecular markers as an aid in the diagnosis of glandular odontogenic cyst. Oral Oncol 2005; 41: 895-902.

12. Tosios KI, Kakarantza-Angelopoulou E, Kapranos N. Immunohistochemical study of bcl-2 protein, Ki-67 antigen and p53 protein in epithelium of glandular odontogenic cysts and dentigerous cysts. J Oral Pathol Med 2000; 29: 139-44. 